\title{
Genève ou les acteurs de la territorialité urbaine
}

\section{Introduction: les fondements de la territorialité}

C'est à partir d'un système de relations entretenu avec son environnement physique et humain que chaque société transforme les ressources dont elle dispose pour créer son territoire. A la fois présentation et représentation du monde, lieu de convergence et de divergence de pratiques consensuelles et marginales, réelles et imaginaires, le territoire est la création jamais finie d'une fusion permanente entre l'extériorité et l'intériorité des rapports de l'homme à la nature et à la culture. Ce système relationnel, d'une extrême complexité, régule les comportements humains en instituant des réseaux d'échange et de communication qui constituent la territorialité, lato sensu, du groupe social concerné. Ces réseaux se rapportent à des stratégies individuelles et collectives qui font l'originalité d'un groupe par rapport à d'autres groupes sociaux. Ainsi, le territoire et la territorialité rendent compte de l'historicité d'une pratique qui consiste en une relation transformée des lieux et des hommes, conception qui renvoie à une compréhension de la pratique dont ils sont les sujets. Si le territoire est le produit de l'ensemble des territorialités, celles-ci n'ont de sens que par rapport au territoire qui les contient. Cette simultanéité fait du territoire un lieu organisé pour organiser, signifié pour signifier et socialisé pour socialiser. Il est un produit social créé sur la base d'un réseau d'échange et de communication médiatisé par un mode de production (MP).

Notre intention est de rendre compte de la territorialité à partir d'un cas concret, celui de l'aménagement urbain en ville de Genève. La présentation des acteurs de la production urbaine a pour objectif de définir le sens des relations qu'ils entretiennent entre eux en tenant compte de leur plus ou moins grande capacité d'influencer les modes d'appropriation et d'aménagement de la cité.

\section{Territorialité et pouvoir}

Une territorialité basée sur des relations dissymétriques implique que l'un des termes de la relation veut obtenir tous les avantages (gains) qui sont l'enjeu de son projet social'. Ses objectifs seront construits autour d'un médiateur que nous définissons, dans ce cas, comme le mode de production à finalité productiviste (MPFP) qui instaure une relation imposant une dépendance de l'un des termes de la relation vis-à-vis de l'autre. Pour un acteur, l'état de dépendance suppose que les coûts relationnels sont plus élevés que les gains, d’où un déficit relationnel. Celui-ci peut provoquer la disparition complète de l'acteur ou du moins sa soumission de plus en plus grande. Dans le cadre de l'appropriation et de l'aménagement du territoire, le MPFP situe la dépendance relationnelle dans le pouvoir décisionnel que possèdent ou non les acteurs en présence. Créé sur une telle base, ce mode tend à évoluer, comme nous avons tenté de le démontrer, indépendamment des intérêts et motivations de la plupart des acteurs ${ }^{2}$. Au contraire, le mode de production à finalité existentielle (MPFE) est un médiateur construit à partir de relations symétriques. Les deux termes de la relation pratiquent une territorialité axée sur des situations d'indépendance dans la mesure où leur dynamique comportementale aboutit à la réalisation partielle ou totale des projets respectifs. Les acteurs, tout en ayant consenti des coûts, sont gagnants, ce qui leur permet de maintenir leur identité et l'équilibre de l'ensemble du système relationnel. Le mode de production représente la somme des relations symétriques et dissymétriques: MP = MPFP + MPFE. Ce qu'il faut donc reconnaître, c'est la qualité de ce mode. Si les relations à partir desquelles se construit le MP sont à prédominance dissymétriques, nous serons en présence du MPFP. Dans le cas contraire, nous serons en présence du MPFE. Pour un acteur, le gain et/ou la perte relationnelle dépendent de sa capacité à contrôler les relations qu'il entretient avec son environnement physique et humain. Toute relation est une combinaison d'énergie et d'information qui constitue un ensemble de signes qui nous renseignent sur la forme et le contenu d'un objet, d'une activité ou d'un message quelconque ${ }^{3}$. La reconnaissance de ces signes permet une interprétation du sens à accorder à cet objet, à cette activité ou à ce message. L'énergie et l'information deviennent ainsi des pro-

Philippe Cordey, Dr., Ecole supérieure de commerce de Genève, 62, rue de Saint-Jean, 1203 Genève. 
duits socio-culturels indissociables d'une certaine représentation de la réalitét. Dans chaque relation, un acteur est à la fois émetteur et récepteur de flux d’énergie et d'information. Le pouvoir contenu dans cette relation vient de la possibilité ou non pour lui de contrôler le sens de ces flux dans le but d'imposer sa propre représentation de la réalité. La territorialité suppose donc un dynamisme dans lequel apparaissent des rapports de force, réseaux conflictuels ou non, créés à partir de l'image que les hommes se font de leur position sociale, dans les limites qu'autorise le système de la répartition des pouvoirs. De là semble-t-il la nécessité, au plan de l'explication, d'une problématique visant à autoriser la mise en perspective du comportement des différents groupes d'acteurs, en cherchant à dégager les motivations qui les guident dans le cadre particulier du mode de production urbaine.

Les acteurs se localisent dans une organisation sociale hiérarchisée en fonction de leur possibilité d'exercer un pouvoir décisionnel dans la production de leur territoire. Le rapport de force apparaît alors de la façon suivante: d'un côté, des décideurs qui cherchent à maintenir et à consolider leur pouvoir de décision, qui leur donne accès, entre autres, au contrôle social; de l'autre côté, des usagers qui ne sont pas ou peu impliqués dans les prises de décision et qui, tout en réfléchissant au sens de leur présence dans l'organisation sociale, tentent de faire valoir leurs intentions et aspirations. En remettant parfois en cause le pouvoir des premiers nommés, ils refusent les processus d'échange et de communication dissymétriques qui leur sont imposés pour exiger de nouvelles formes relationnelles plus égalitaires, plus autonomes.

Sur le plan politique, le rôle des institutions consiste à gérer les systèmes relationnels qui s'établissent entre tous les acteurs et groupes d'acteurs qui participent à la création de leur territoire. L'Etat est le régulateur de la production sociale. Son pouvoir s'exprime par l'intermédiaire de normes (la Constitution, l'appareil législatif, etc.) reconnues par tous et considérées comme des médiateurs garants de la stabilité et du maintien des institutions. En Suisse, l'espace normé, qu'il soit constitutionnel ou autre, relève d'une attitude consensuelle très marquée qui renforce le rôle de filtre idéologique de l'Etat. Etendu à tous les niveaux (fédéral, cantonal et communal), le consensus empêche la remise en cause du fédéralisme par intégration de la plupart des options idéologiques à l'élaboration du productivisme helvétique. Les processus d'échange et de communications se réalisent à l'intérieur d'espaces normés sur le plan social, économique, politique et culturel qui représentent autant de lieux d'affirmation et de reconnaissance de l'autorité. Ce sont aussi les lieux dans lesquels apparaissent les refus, politisés ou non, des attitudes consensuelles.

\section{Les acteurs de la territorialité urbaine à Genève}

Depuis 1945, l'extension urbaine est liée à l'essor économique du canton. Les activités bancaires et commerciales, les organisations internationales rendent attractive la région genevoise qui recevra un apport considérable de population confédérée et étrangère (près de 30000 personnes entre 1950 et 1960). La tertiarisation du centre-ville et l'arrivée de nouveaux habitants obligent à l'extension extramuros de l'habitat en direction des quartiers périphériques au centre historique (Champel, Malagnou, Florissant, Grand-Pré, etc.) d'une part, des plus proches campagnes d'autre part. Sur ces dernières s'érigeront successivement à partir des années soixante et jusqu'au milieu des années septante, des grands ensembles (Le Lignon, La Gradelle, Les Avanchets) et des cités-satellites (Lancy-Onex, Meyrin) $)^{5}$. Des locatifs plus modestes en taille seront ensuite construits sur l'ensemble du canton (Bernex, Puplinge, Chêne-Bourg, Vernier, etc.) en même temps que la mise en valeur des noyaux villageois. C'est à partir de la fin des années septante qu'apparaît la reconquête, en ville de Genève, des quartiers anciens. Changement fonctionnel et densification de l'habitat surtout caractérisent cette rénovation, véritable opération d'assainissement qui permet le retour intra-muros d'une partie de la population qui accepte de payer parfois fort cher le droit de retrouver la centralité urbaine.

Dans la réalité sociale, chacun des termes de la relation (émetteur et/ou récepteur) est une représentation d'acteurs ou de groupes d'acteurs entre lesquels la complexité de l'échange varie en fonction de l'enjeu qui conditionne leur mise en présence. Nous nous proposons donc de présenter les motivations qui les guident dans le cadre particulier de la production urbaine. Bien qu'issue de nos observations genevoises, la présentation qui suit se veut de portée générale.

\section{L'Etat}

Le développement urbain genevois reflète des stratégies d'appropriation et d'aménagement du territoire qui se déploient en fonction des nécessités socio-économiques et démographiques. Sur le plan politique, il est l'objet d'un rapport de force entre différents groupes d'acteurs et médiatisé par l'Etat dont la fonction est double: d'une part il se comporte comme un agent régulateur du marché immobilier qui, sur la base d'un appareil législatif, oblige les acteurs à tenir compte de certaines normes conciliables avec les intérêts de la société. D'autre part, il se comporte comme n'importe quel acteur immobilier qui achète, vend, construit ou démolit des terrains ou des immeubles. 


\section{Les décideurs-aménageurs}

En raison des moyens financiers dont ils disposent et des relations qu'ils entretiennent avec les autorités politiques, ces acteurs développent des stratégies qui modèlent la forme et le contenu du territoire urbain. Leur action se concrétise, dans un premier temps, par une appropriation juridique, momentanée ou définitive, pour rendre le territoire, dans un second temps, propre à un usage particulier. Ils décident (pôle émetteur) de l'orientation de la production sociale en accord avec l'Etat qui régule leur activité à l'aide de règles issues de la politique consensuelle. Ce groupe d'acteurs rassemble les entreprises publiques et privées suivantes: les banques, les compagnies d'assurance, les établissements hypothécaires, les syndicats patronaux et ouvriers ainsi que la plupart des grandes entreprises commerciales, industrielles et financières. Leur poids décisionnel est important dans la mesure où la détention du capital leur permet d'agir sur la politique économique et sociale. Dans le domaine de l'édification du bâti urbain, ces entreprises agissent de plusieurs façons: soit directement en tant que propriétaire, promoteur ou constructeur, en se fournissant mutuellement les capitaux nécessaires; soit indirectement par des aménageurs-exécutants (voir ci-dessous) qui utilisent le capital mis à leur disposition (crédits à la construction par exemple). D'autres organisations publiques et privées jouent un rôle non négligeable dans le domaine immobilier: les fonds de prévoyance, les caisses de retraites ou les institutions religieuses participent à des programmes de construction et sont souvent eux-mêmes propriétaires. Enfin, les spéculateurs sont des décideurs-aménageurs parce qu'ils offrent aux acteurs de la catégorie à laquelle ils appartiennent (et qui peuvent aussi spéculer) des lieux d'action selon un travail de prospective et une stratégie qui les font décider, à un moment donné, de mettre sur le marché des terrains qui peuvent être équipés. Leur pouvoir décisionnel est important, même si leur poids politique et financier ne l'est pas autant.

\section{Les aménageurs-exécutants}

Si l'appropriation du territoire relève essentiellement des décideurs-aménageurs et de l'Etat, ce sont les aménageurs-exécutants qui sont responsables de son aménagement. Aménager consiste à mettre en place une organisation de la population, des activités et des lieux en vue de réaliser des objectifs politiques, socio-économiques et culturels. Pôle récepteur des décisions prises par les décideurs-aménageurs, les aménageurs-exécutants émettent un savoir-faire qui les rend indispensables:

le promoteur est celui qui met en marche le processus de construction et qui coordonne toutes les activités de la phase constructive. Il réunit un capital qu'il transforme en valeur marchande et qui, une fois la construction terminée, se transforme à nouveau en capital ${ }^{6}$;

l'architecte procède de deux façons: premièrement, il exécute des commandes imposées par les décideurs-aménageurs (fonction d'exécution); deuxièmement, il soumet des projets lui permettant dexprimer plus librement sa créativité au service d'intérêts publics et privés (fonction de création):

les sociétés immobilières apportent des capitaux pour un programme précis de construction et ne s'occupent, le plus souvent, que de la gestion de l'immeuble qui vient d'être construit. Leur domiciliation est souvent chez les régies;

le régisseur fait de la gestion d'immeubles et parfois de la promotion. Dans ce dernier cas, en s'inscrivant comme régisseur, le promoteur peut participer à la création de sociétés immobilières:

les sociétés coopératives de construction sont constituées par les futurs occupants de l'immeuble ou groupe d'immeubles. Ce sont surtout les syndicats qui utilisent cette forme juridique pour intervenir directement dans la production urbaine;

l'ingénieur ou autre technicien, par son haut degré de qualification, maîtrise les procédés techniques dans l'élaboration du domaine bâti. Il peut être indépendant ou appartenir à des entreprises générales de construction;

les entreprises générales de construction participent à tous les stades de l'édification du bâti urbain. Elles font de la promotion, elles possèdent des bureaux d'ingénieurs et d'architectes, elles construisent, vendent et louent les surfaces ainsi produites;

les entreprises de construction interviennent uniquement dans la phase de construction.

Le propriétaire foncier n'est pas un acteur en luimême. Certes, la possession d'un terrain ou d'un immeuble, grâce à la rente foncière, permet d'exercer une pression sur le marché immobilier. Cependant, la construction et les opérations qui s'ensuivent sont plus importantes que la détention d'un terrain. Un conflit peut alors apparaître entre un propriétaire qui a reçu un terrain ou un immeuble en héritage ou qui a pu l'acheter par le rendement de son travail, et une entreprise qui désire acquérir des parcelles en vue d'une opération immobilière. Les décideurs-aménageurs et leurs exécutants sont les moteurs de la croissance économique. Entre eux, les relations se créent sur des bases d'affaires, familiales ou politiques. La densité des liens ainsi crées favorise la coordination et le contrôle de leurs actions.

\section{Les usagers}

Ils représentent la grande majorité des acteurs qui ne participent pas aux décisions concernant l'appropriation et l'aménagement du territoire. Ils se divi- 
sent en deux catégories: les propriétaires (d'un immeuble, d'un appartement) et les locataires. C'est à partir de la fin des années soixante que certains groupes d'usagers s'organisent et se font connaître sur la scène du théâtre urbain genevois. Opposés à une tertiarisation excessive qui transforme morphologiquement et fonctionnellement la cité, ils s'affrontent aux milieux immobiliers et à l'Etat à travers des actions plus ou moins politisées selon les cas. Récepteurs des stratégies imposées par les groupes d'acteurs précédents, les usagers possèdent cependant les moyens de s'y opposer: la pétition, les droits de référendum et d'intitative ainsi que le droit de recours auprès des commissions étatiques leur permettent de jouer un rôle parfois décisionnel, mais surtout régulateur. Avec l'utilisation de tels moyens, ils représentent une première forme de résistance et se regroupent de la façon suivante $e^{7}$ :

les groupes spontanés interviennent dans des lieux bien localisés: les squatters, les groupes référendaires sont des exemples de regroupements spontanés pour répondre à des préoccupations conjoncturelles;

les associations de locataires agissent sous la forme d'un syndicalisme de consommation du logement autour de la question du contrat de bail locatairepropriétaire;

les associations de défense du patrimoine ne défendent ni un lieu ni une relation économique, mais une image. Leur intervention se situe au-delà des contingences socio-économiques par une sensibilisation à l'image de la ville, à la forme architecturale, aux lieux historiquement reconnus (par exemple, la Société d'art public, Sauvegarde et Progrès, le Guet).

En dehors de ces formes de regroupement aparaissent des usagers qui dénoncent l'inégalité d'accès aux modes d'appropriation et d'aménagement du territoire. Ils développent une argumentation critique dans le but de refuser les processus qui empêchent les usagers de participer à l'élaboration de leur cadre de vie. Ainsi apparaît la lutte urbaine, définie comme un rapport de pouvoir qui s'établit entre des groupes d'usagers et les institutions politicoéconomiques. Cette remise en cause de la production urbaine se généralise à l'échelle de la société dans le but de repenser les rapports de l'homme à son environnement physique et humain à partir des lieux même d'émergence de ces rapports:

les groupes de quartier représentent une seconde forme de résistance, plus agressive et politisée. Nés dans la foulée des événements de 1968, ils interviennent pour la défense de l'habitat et l'amélioration des conditions de vie en ville (par exemple: le Cartel des Pâquis, l'Action populaire aux Grottes, le Groupe logement Plainpalais ou encore, le plus ancien, le Groupe d'habitants des Eaux-Vives). Sans avoir la prétention d'être les seuls acteurs du changement social, leur projet consiste à développer des stratégies qui soient représentatives des préoccupations réelles des usagers.

Les migrations extra ou intra-muros des habitants dépendent de l'importance du déploiement des activités en général, des activités tertiaires en particulier dans le cas genevois. Depuis 1950, trois phases de tertiarisation ont provoqué sur le bâti urbain des transformations morphologiques et fonctionnelles par des interventions par rue massives ou diffuses selon les époques. Ces trois phases correspondent à autant de comportements différents de la part des usagers face aux décideurs-aménageurs et à leurs exécutants:

\section{1) tertiarisation centrale et rénovation massive:}

jusqu'au milieu des années septante, le déploiement des activités de services réduit très fortement la fonction résidentielle du centre-ville historique et de ses quartiers périphériques. C'est pendant cette période que les usagers se regroupent pour lutter contre la rénovation massive qui détériore la qualité de leurs lieux de vie ou les rejette dans les communes périphériques à la commune Ville de Genève. La contestation atteint son point culminant pendant les années septante avec la lutte des groupes de quartier qui sont les seuls à s'opposer efficacement à la destruction de l'habitat;

2) tertiarisation centrale et périphérique et rénovation diffuse:

l'exiguité du territoire urbain limite l'extension des activités de services. A partir du milieu des années septante, celles-ci trouvent de nouveaux terrains d'expansion dans les quartiers situés en périphérie de la commune Ville de Genève ou dans les zones industrielles et commerciales du canton. Cette nouvelle orientation de l'aménagement du territoire modifie profondément les relations entre les différents groupes d'acteurs. La rénovation diffuse qui, à partir des années quatre-vingt, caractérise le mode interventionniste des milieux immobiliers et de l'Etat met en valeur d'autres formes de résistances: les groupes spontanés et les associations de défense du patrimoine prennent le relais des groupes de quartier. En agissant sur le terrain de l'action ponctuelle, ils créent de nouveaux rapports de force, moins politisés.

3) tertiarisation périphérique et rénovation diffuse: l'occupation des zones industrielles et commerciales continue parallèlement au développement de prestations de services «haut de gamme» dans la zone aéroportuaire de Cointrin qui concurrence très fortement les activités du centre-ville historique. Al'intérieur de la cité, la tertiarisation est de plus en plus diffuse et la rénovation de plus en plus ponctuelle. Actuellement, la problématique qui conditionne les modes d'appropriation et d'aménagement du territoire n'est plus fondée sur l'unique déploiement 
quantitatif des activités. Des préoccupations plus qualitatives, écologiques, induisent des attitudes nouvelles parmi les décideurs et les usagers qui n'agissent plus sur le terrain de la confrontation frontale plus ou moins violente. Entre les trois phases présentées, une nouvelle génération d'acteurs est apparue en même temps que changeaient les conditions du développement économique. L'exiguité du territoire genevois ainsi que les réelles préoccupations au sujet de la qualité de la vie obligent à des attitudes plus consensuelles qui changent complètement le sens de la résistance des usagers. Celle-ci, plus discrète, n'est plus le privilège de tel ou tel groupe d'acteurs.

\section{Conclusion: pour une géographie de la territorialité}

La géographie de la territorialité explique la production sociale sous la forme d'un enjeu entre les hommes qui s'affrontent sur le terrain de l'appropriation et de l'aménagement du territoire pour y construire des lieux de vie. Les décideurs-aménageurs et leurs exécutants accordent une valeur d'échange à ce territoire considéré comme une marchandise. Les revendications des usagers, lorsqu'elles existent, accordent une valeur d'usage à ce territoire considéré comme un lieu d'expression et d'affirmation d'identités individuelles et collectives. En privilégiant une observation à grande échelle, la géographie de la territorialité met en valeur des faits et des vécus qui permettent la mesure des écarts entre les perceptions d'échange et d'usage privilégiées par les différents groupes d'acteurs.

La découverte de la territorialité s'inscrit dans un mouvement général d'enrichissement des problématiques des sciences sociales ayant découvert la nécessité de mettre en perspective réciproque de multiples projets sociaux pour comprendre les systèmes relationnels liant les hommes à leur environnement physique et humain. Elle rend ainsi compte d'une analyse à grande échelle des relations humai- nes pour aboutir au sens de l'organisation du territoire à partir du pouvoir qui caractérise les groupes d'acteurs en présence. Ce type d'analyse cherche à rendre explicite ce que les acteurs cherchent implicitement dans les relations qu'ils entretiennent entre eux. La finalité, c'est la mise en place d'une théorie scientifique de ce dont les gens ont d'expérience quotidienne et qui restitue le travail accompli par chacun dans un contexte macroscopique. Sur le plan urbain, l'observation à l'échelle des acteurs est un moyen de faire comprendre qu'une ville ne peut se figer dans des formes auxquelles la société aurait attribué certaines valeurs. Elle est, au contraire, le lieu d'une aventure sociale à travers laquelle se réalise une expression anthropologique des sociétés ${ }^{8}$.

\section{Notes}

1 Voir à ce sujet les écrits de CL. RAFFESTIN:

- Pour une géographie du pouvoir (LITEC; Paris, 1980).

- "Paysage et territorialité" (Cahiers de Géographie du Québec, vol. 21, pp. 123-134, 1977).

2 Voir PH. CORDEY:

- Appropriation et aménagement du territoire: Genève, essai d'écologie urbaine (PETER LANG AG, Bern, 1984).

- "Pour une géographie écologique: interprétation d'une mise en jeu des acteurs à travers l'analyse des coûts sociaux de la rénovation urbaine" (Cahiers de Géographie du Québec, vol. 29, no 78, pp. 405-413, 1985).

3 J. ATTALI, La parole et l'outil (PUF, Paris, 1976).

${ }^{4}$ Ibid.

5 Créations rendues possibles par les lois HLM ainsi que par la loi sur l'expansion de l'agglomération urbaine de 1957.

$6 \mathrm{CH}$. TOPALOV, Les promoteurs immobiliers (Mouton, Paris, 1979).

7 Voir à ce sujet PH. CORDEY, PH. GFELLER, J. MARTIN, "Les luttes urbaines à Genève" (Revue suisse de sociologie, vol. 12, no 3, pp. 451-469, 1986).

8 Voir A. MÉDAM, Conscience de la ville (Anthropos, Paris, 1976). 


\section{Literaturbesprechung}

CARRON Alexandre/Christophe: Nos cousins d'Amérique. Histoire de l'émigration valaisanne au XIXe siècle. Collection Mémoire Vivante, $300 \mathrm{~S}$., zahlr. Abb., Monographic Sierre, 1986.

On sait que les crises du $X X^{e}$ siècle ont amené des millions d'Européens à s'expatrier en quête de la fortune sur d'autres continents. On sait que les Suisses ont fait partie en nombre de ces convois impatients de coloniser le monde. Des historiens ont raconté les aventures peu banales de ces compatriotes séduits par les mirages des eldorados américains. Cette histoire s'enrichit aujourd'hui d'un nouveau chapitre, celui des Valaisans émigrés en Argentine. Et l'on découvre avec étonnement qu'au pays du tango, les Bonvin, Morard, Bruchez, Delaloye et autres Biderbos représentent de nombreuses familles installées dans des colonies qui ont conservé un caractère valaisan bien marqué.

"Nos Cousins d'Amérique" est l'ouvrage qui manquait au Valais sur l'émigration de ses compatriotes. Il est le résultat d'un long travail de recherches d'une quinzaine d'années au cours desquelles plusieurs voyages en Amérique et de nombreuses relations entretenues de pat et d'autre de l'Atlantique on permis aux auteurs de rassembler une documentation riche et variée: lettres, carnets de voyage, témoignages, photographies, contrats de colonisation etc. Ces documents ont utilement complété les sources des archives cantonales, assez lacunaires en la matière, et sont abondamment reproduits dans l'ouvrage des Carron.

On apprend dans ce livre que de 1850 à 1914 pas moins de 18000 individus ont préféré tenté leur chance outre-mer plutôt que de végéter au pays. 85 \& de ces émigrants ont choisi l'Amérique du sud, en grande majorité l'Argentine, mais aussi le Brésil et l'Uruguay, le reste s'installant aux USA et au Canada.

Alexandre et Christophe Carron analysent la situation en Valais au XIX ${ }^{e}$ siècle, les conditions particulières qui expliquent les nombreux départs assez constants de 1850 à 1900, les difficiles expériences faites par les pionniers. Ainsi les premières vagues d'émigration vers l'Algérie ou l'Amérique du Sud dans les années 1850 se soldent-elles par des échecs. Puis les émigrants, au prix de mille souffrances, ouvrent le chemin a leurs compatriotes qui les rejoignent plus tard et, forts des expériences faites, bâtissent sur des fondements solides.

A San José et dans les colonies d'Espéranza et de San Géronimo en Argentine, les Valaisans furent les pionniers de l'agriculture intensive et extensive. Ils furent les pionniers de l'industrie laitière, selon les traditions alpines, exploitant en particulier la fabrication du beurre et ils furent les pionniers de la subdivision des terres. Ils ont su se créer une place en Argentine, faire prospérer leurs entreprises, offrir une patrie a leurs enfants.

C'est l'histoire de ces familles et leur épopée que se propose ce livre. Les héros n'ont pas manqué, connus ou anonymes, dans cette oeuvre colonisatrice où le rêve a tenu parfois une place éxagérée. Si la réalité a tôt dessillé les yeux éblouis de certains émigrants, l'aventure a parfois dépassé le rêve en offrant la fortune et la célébrité à des hommes remarquables qui ne croyaient pas trouver dans leur patrie un champ d'action à la mesure de leur énergie et de leurs talents.

Michel Roten, Sion 